\title{
Right-sided Colectomies for Diverticulitis Have Worse Outcomes Compared to Left-Sided Colectomies for Diverticulitis: An ACS NSQIP analysis of Predictors and Outcomes
}

Nathalie Wong-Chong, Nancy Morin, Gabriela Ghitulescu, Carol-Ann Vasilevsky, Philip Gordon, Julio Faria, Marylise Boutros

Division of Colon and Rectal Surgery, Sir Mortimer B. Davis Jewish General Hospital, Montreal, QC, Canada

\section{Introduction}

- Right- and left-sided diverticulitis have similar clinical presentations

- Right-sided diverticulitis tends to affect younger patients, usually of Asian heritage

- There are limited and conflicting data on the surgical outcomes following resection for right-sided compared to left-sided diverticulitis.

\section{Purpose}

- The aim of this study was to compare short-term outcomes of colectomies for right- and left-sided diverticulitis.

\section{Methods}

- After institutional review board approval, all cases of right- and left-sided colectomy for diverticulitis were identified from the American College of Surgeons National Surgical Quality Improvement Program (NSQIP) database from 2005-2015

- Patients with diverticulitis were identified based on ICD-9 diagnostic codes (ICD-9 562.11, 562.13)

- Right- and left-sided colectomy were identified by primary procedure CPT codes

- Demographics, comorbidities, and postoperative outcomes were identified and compared

- Predictors of the pre-defined outcomes were analyzed by multivariate regression

\section{Results}

Table 1. Patient Characteristics (50,288 patients)

\begin{tabular}{|c|c|c|c|}
\hline $\begin{array}{l}\text { Characteristic } \\
\mathrm{N}(\%) \text { or Mean (SD) }\end{array}$ & $\begin{array}{l}\text { Right-sided } \\
\text { colectomy } \\
(n=710)\end{array}$ & $\begin{array}{l}\text { Left-sided } \\
\text { colectomy } \\
(n=49,878)\end{array}$ & $\begin{array}{l}p- \\
\text { value }\end{array}$ \\
\hline Age & $55.98(14.68)$ & $58.45(13.07)$ & $<0.01$ \\
\hline $\begin{array}{l}\text { Race } \\
\text { Asian }\end{array}$ & $26(3.66)$ & $416(0.85)$ & $<0.01$ \\
\hline $\begin{array}{l}\text { BMI } \\
\begin{array}{l}<18 \\
18.0-24.9 \\
25-29.9 \\
30.0-34.9 \\
>35.0\end{array}\end{array}$ & $\begin{array}{l}24(3.38) \\
216(30.42) \\
236(33.24) \\
153(21.55) \\
81(11.41)\end{array}$ & $\begin{array}{l}1,210(2.46) \\
11,340(23.08) \\
17,173(34.94) \\
11,321(23.04) \\
8,100(16.48)\end{array}$ & $<0.01$ \\
\hline $\begin{array}{l}\text { Smoker } \\
\mathrm{CHF}\end{array}$ & $\begin{array}{l}176(24.79) \\
10(1.41)\end{array}$ & $\begin{array}{l}10,632(21.63) \\
333(0.68)\end{array}$ & $\begin{array}{l}0.04 \\
0.02\end{array}$ \\
\hline Bleeding disorder & $46(6.48)$ & $1860(3.78)$ & $<0.01$ \\
\hline $\begin{array}{l}\text { Preoperative blood } \\
\text { transfusion }\end{array}$ & $23(3.24)$ & $298(0.81)$ & $<0.01$ \\
\hline Emergency surgery & $168(23.66)$ & 7654 (15.57) & $<0.01$ \\
\hline Laparoscopy & $356(50.14)$ & $26,837(54.61)$ & 0.02 \\
\hline $\begin{array}{l}\text { Preoperative Sepsis } \\
\text { None } \\
\text { SIRS } \\
\text { Sepsis } \\
\text { Septic Shock }\end{array}$ & $\begin{array}{l}590(83.10) \\
42(5.92) \\
73(10.28) \\
3(0.42)\end{array}$ & $\begin{array}{l}41,999(85.46) \\
2038(4.15) \\
4483(9.12) \\
525(1.07)\end{array}$ & $<0.05$ \\
\hline Stoma & $4(0.56)$ & $10,515(21.40)$ & $<0.01$ \\
\hline
\end{tabular}

\section{Results}

Table 2. 30-day Post-operative Outcomes

\begin{tabular}{llll}
\hline $\begin{array}{l}\text { Outcome } \\
\text { N (\%) or Mean (SD) }\end{array}$ & $\begin{array}{l}\text { Right-sided } \\
\text { colectomy } \\
(\mathrm{n}=710)\end{array}$ & $\begin{array}{l}\text { Left-sided } \\
\text { colectomy } \\
(\mathrm{n}=49,878)\end{array}$ & $\begin{array}{l}\boldsymbol{p} \text { - } \\
\text { value }\end{array}$ \\
\hline $\begin{array}{l}\text { SSI } \\
\quad \text { Superficial }\end{array}$ & $48(6.76)$ & $3391(6.90)$ & 0.88 \\
$\quad$ Deep & $9(1.27)$ & $641(1.30)$ & 0.93 \\
$\quad$ Organ/Space & $41(5.77)$ & $2062(4.20)$ & 0.04 \\
\hline Sepsis & $61(8.59)$ & $3586(7.30)$ & 0.19 \\
\hline Bleeding/Transfusion & $47(6.62)$ & $2273(4.63)$ & 0.01 \\
\hline 30-Day Major Morbidity & $166(23.38)$ & $10,157(20.67)$ & 0.07 \\
\hline 30-Day Mortality & $13(1,83)$ & $624(1.27)$ & 0.19 \\
Reoperation & $51(7.18)$ & $2354(4.79)$ & $<0.01$ \\
\hline Readmission & $48(11.03)$ & $2733(8.37)$ & $<0.05$ \\
\hline Length of stay (days) & $8.5(8.7)$ & $7.4(7.46)$ & $<0.01$
\end{tabular}

Table 3. Colectomy-specific 30-day Post-op Outcomes

\begin{tabular}{llll}
\hline $\begin{array}{l}\text { Outcome } \\
\text { N (\%) }\end{array}$ & $\begin{array}{l}\text { Right-sided } \\
\text { colectomy } \\
(\mathrm{n}=214)\end{array}$ & $\begin{array}{l}\text { Left-sided } \\
\text { colectomy } \\
(\mathrm{n}=15,085)\end{array}$ & $\begin{array}{l}\boldsymbol{p} \text { - } \\
\text { value }\end{array}$ \\
\hline Anastomotic Leak & $12(9.81)$ & $438(2.90)$ & $<0.01$ \\
\hline $\begin{array}{l}\text { Management of Leak } \\
\quad \text { Reoperation }\end{array}$ & $6(2.34)$ & $245(1.62)$ & $<0.01$ \\
$\quad$ Percutaneous drain & $5(2.34)$ & $95(0.63)$ & \\
$\quad$ Non-operative & $1(0.47)$ & $61(0.40)$ & \\
$\quad$ No intervention & 0 & $37(0.25)$ & \\
$\quad$ Unknown & $3(1.40)$ & $55(0.36)$ & \\
Postoperative lleus & $36(16.82)$ & $1798(11.92)$ & 0.07
\end{tabular}

Table 4. Multivariate analysis for right-sided colectomy*

\begin{tabular}{lll}
\hline & OR & $\mathbf{9 5 \%} \mathbf{C l}$ \\
\hline Anastomotic leak & 2.04 & $1.13-3.68$ \\
Major Morbidity & 1.31 & $1.06-1.63$
\end{tabular}

*After controlling for emergency surgery, ASA, immunosuppression, and sepsis amongst other confounders

- Stomas were protective against anastomotic leak (OR $0.38,95 \% \mathrm{Cl} 0.26-0.55)$ for both right- and left-sided colectomy

- Emergency surgery was also a predictor for major morbidity (OR $1.42,95 \% \mathrm{Cl} 1.53-2.66)$ for both right and left-sided colectomies.

- Type of colectomy (right vs. left-sided) was not a predictor of mortality

\section{Conclusions}

- Re-operation and readmission rates were higher with right-sided colectomy

- Even after controlling for emergency surgery, right-sided colectomy was still an independent risk factor for anastomotic leak, length of stay and major morbidity.

- Stomas were protective against anastomotic leak, but very few cases of right-sided diverticulitis were diverted with an ileostomy.

- Further research required to determine whether morbidity associated with right-sided colectomies could be reduced with more frequent use of diverting stomas. 